\title{
Lidil
}

Revue de linguistique et de didactique des langues

\section{Pour une approche typologique des prédicats d'état en russe et en français}

Le cas des adjectivaux

\section{Irina Kokochkina}

\section{(2) OpenEdition}

\section{Journals}

Édition électronique

URL : http://journals.openedition.org/lidil/2696

DOI : $10.4000 /$ lidil.2696

ISSN : 1960-6052

Éditeur

UGA Éditions/Université Grenoble Alpes

Édition imprimée

Date de publication : 1 juin 2008

Pagination : 133-148

ISBN : 978-2-84310-124-3

ISSN : $1146-6480$

Référence électronique

Irina Kokochkina, «Pour une approche typologique des prédicats d'état en russe et en français », Lidil [En ligne], 37 | 2008, mis en ligne le 01 septembre 2009, consulté le 01 mai 2019. URL : http:// journals.openedition.org/lidil/2696 ; DOI : 10.4000/lidil.2696 


\title{
POUR UNE APPROCHE TYPOLOGIQUE DES PRÉDICATS D'ÉTAT EN RUSSE ET EN FRANÇAIS
}

\author{
LE CAS DES ADJECTIVAUX
}

\author{
Irina KOKOCHKINA*
}

\begin{abstract}
RÉSUMÉS
Le présent article est consacré aux prédicats adjectivaux d'états en russe et en français. Nous proposons un faisceau de paramètres qui permettent de décrire ces prédicats (l'interrogation, l'intensité, le temps et l'aspect, etc.) et nous décrivons en détail certains d'entre eux. La comparaison des deux langues, l'une slave et l'autre romane, démontre la validité des paramètres d'analyse proposés. Bien que l'article soit consacré aux prédicats adjectivaux, ladite méthode pourrait être utilisée pour décrire tous les états (prédicats adjectivaux, nominaux ou verbaux).
\end{abstract}

\section{ABSTRACT}

This paper is about predicates of state [nредикат состояния] expressed by adjectives, in Russian and in French. We propose a list of parameters facilitating description of several types of predicates, such as types of questions, intensity, tense and aspect, cause, etc. We also describe in details some of these predicates. The comparison between the two languages, a Slavic and a Romance one, demonstrates the validity of the proposed parameters. Whilst adjectives are central in the analysis undertaken here, the criteria drawn upon could be used to build a new typology of all predicates of state.

Notre étude porte sur les propriétés sémantiques et syntaxiques des prédicats adjectivaux généralement regroupés sous le terme d' «états». Après avoir défini notre objet de recherche, nous essaierons de démontrer que tous les états peuvent être décrits au moyen d'un faisceau de paramètres

*Université Paris-Sorbonne 
sémantiques et syntaxiques communs. En prenant comme exemple quelques-uns de ces paramètres, nous essaierons de montrer que la méthode d'analyse choisie nous permet de classer les adjectifs d'états et ce, dans les deux langues, et aboutit, en définitive, à une typologie basée sur des critères stricts. La démarche contrastive nous permettra de vérifier la validité de la démarche adoptée.

\section{Remarques préliminaires}

La notion d'état a suscité une abondante bibliographie. Tout d'abord, il convient de signaler les recherches qui visent à définir globalement les états par une série de traits sémantiques $^{1}$. Il faut ensuite mentionner ceux qui traitent de telle ou telle classe sémantique spécifique. Mais aucun de ces travaux n'a de visée typologique. Sans prétendre à une analyse détaillée de la structuration interne des «états», nous avons choisi d'étudier les adjectivaux ${ }^{2}$, que nous définirons de la façon suivante: NO être Pred. en français et NO byt' Pred. en russe (où $N O$ renvoie au sujet et $P r e d$. au prédicat adjectival) ${ }^{3}$.

1. Ces travaux se basent souvent sur la classification de $Z$. Vendler (1967). Ainsi, T.V. Bulygina (1982) et E.V.Padučeva (1996) définissent les états en russe par les traits suivants: stativité, durativité et absence de contrôle. D'autres chercheurs (J.E. Miller, 1970, pour le russe et M.Riegel,1985, pour le français) prennent comme point de départ les critères établis par G. Lakoff (1966): les verbes statifs ne peuvent pas être mis à l'impératif, ils n'acceptent pas les adverbes de manière, etc. Pour le compte rendu détaillé des études sur le russe, nous renvoyons à F. Akmalova (2005) et pour le français, à I. Kokochkina (2004).

2. Ce choix est motivé par le fait qu'en russe, les verbes d'états sont plus nombreux qu'en français. Par ailleurs, beaucoup de travaux sont consacrés aux verbes d'états et à la catégorie d'état.

3. Ce besoin de partir d'une définition formelle est basé sur le constat d'après lequel l'ensemble des états regroupe deux-cent cinquante classes sémantiques différentes et que chacune d'elles doit être définie séparément (Kokochkina, 2004). Voici quelques exemples en français: les prédicats de <couleur $>$ rouge, de $<$ forme $>$ ovale, les $<$ phases d'existence>: en décadence, en ruine, les <états de vie ou de mort>: mort, vivant, les <états de santé> sain, etc. Nous utilisons les critères des adjectifs prédicatifs tels qu'ils sont proposés par G. Gross (1991, 
Quant au cadre théorique, il est défini par les travaux de Zellig Harris. Notre unité minimale d'analyse est la phrase simple. Celle-ci est définie comme l'ensemble du prédicat et des arguments qu'il sélectionne. Par exemple:

(1) Mixail bol'šoj [Michel est grand]. Michel est grand ${ }^{4}$.

Le prédicat (bol'šoj, grand) possède ici un seul argument ${ }^{5}$ représenté par un substantif qui vérifient le trait «humain» (Mixail, Michel).

\section{Les paramètres d'analyse des états}

Pour décrire les états «de l'intérieur», nous avons élaboré une grille de paramètres qui peut être appliquée à la totalité des états et qui permet de systématiser leur description sans la

1996b: 90) : ils peuvent être attributs, ils sont pronominalisables par le, ils ont des degrés de comparaison, le comparatif et le superlatif. Par exemple: blanc, en transe, en plein désespoir, dans le doute, etc. Pour ce qui est du russe, nous suivons T.V. Bulygina (1982: 34-35), qui range les suites du type $v$ vostorge [ravi], $v$ otčajanie [au désespoir], etc. parmi les prédicats d'états.

4. Le statut syntaxique de l'attribut du sujet est un problème ouvert. Ainsi, M. Riegel et al. (1994: 233) le considèrent comme «le deuxième constituant d'un groupe verbal (GV $\rightarrow V+X)$ dont le verbe introducteur est le verbe être ou un verbe d'état susceptible de lui être substitué ». L'attribut est dit «complément du verbe» par M. Wilmet (2003: 508). Notre étude s'inscrit dans un cadre différent, défendu notamment par $\mathrm{L}$. Tesnière (1959: 156), pour qui l'adjectif attribut joue le même rôle structural qu'un verbe monovalent (à un seul actant) et par P. Le Goffic, d'après qui, dans la structure être Adj., il n'y a qu'un seul actant, qui est le siège de l'attribution (1993: 196). D'après les linguistes russes, bolšoj [grand] dans Mixail bol'šoj [Michel est grand] est analysé tantôt comme partie constituante du prédicat nominal composé («sostavnoje imennoe skazujemoje», voir par exemple Valgina), tantôt comme prédicat logique («predikat», voir notamment Bulygina, 1982). C'est ce deuxième point de vue que nous adoptons dans notre étude.

5. Les prédicats d'états peuvent avoir un ou deux arguments. L'argument-sujet est obligatoire, alors que la possibilité d'avoir un deuxième argument est conditionnée par le sémantisme du prédicat. Par exemple, fatigué de qqch., fier de qqch. 
simplifier. Nous allons présenter certains ${ }^{6}$ de ces paramètres : l'interrogation, l'intensité et enfin, le temps et l'aspect. Il ne s'agit pas ici d'en donner une présentation exhaustive (compte tenu des contraintes d'espace), mais de mettre en évidence le principe même d'analyse.

\section{L'interrogation}

Parmi tous les paramètres, l'interrogation mérite une attention particulière, et ce pour deux raisons. Tout d'abord, à notre connaissance, elle n'a jamais été mentionnée dans les études sur les états. Ensuite, elle s'avère extrêmement importante, ce que nous allons essayer de démontrer.

Le paramètre de l'interrogation permet de distinguer quelques grands ensembles, des hyperclasses d'adjectifs d'états. Il y a d'abord les prédicats qui répondent à la question comment ? (kakoj? [comment ?] en russe) et qui servent à décrire leur référent de divers points de vue. C'est notamment le cas des prédicats dits de propriétés, qui renvoient à la couleur, la longueur, la taille, etc. d'un objet ou au caractère, au comportement, etc. d'un sujet humain. Voici quelques exemples en français:

(2a) Comment est ta nouvelle maison? - Elle est (grande + à trois étages).

(2b) Kakoj Vanja ? - (Dobryj + otzyvčivyj + sderžannyj). [Comment est Vania ? - Il est (bon + compatissant + réservé).]

Le paramètre de l'interrogation a pour fondement le phénomène de la référence. Il est bien connu que l'adverbe interrogatif comment? et le pronom-adjectif interrogatif kakoj ? interrogent sur les qualités des sujets humains ou des objets ${ }^{7}$. Ce constat propre aux deux langues demande néanmoins une précision concernant le russe. Contrairement au français, où

6. Les paramètres d'analyse des états sont décrits en détails dans I Kokochkina (2004).

7. Ainsi, d'après I. Bartning (1985: 198), la question Comment ? vise la fonction caractérisante de l'adjectif. 
comment possède une portée assez générale, son équivalent russe, kakoj ? permet uniquement comme réponse les prédicats qui renvoient à une caractéristique inhérente de 1'individu. Il peut s'agir du caractère de la personne (comme dans l'exemple ci-dessus) ou de son comportement. Les autres prédicats descriptifs (ceux qui renvoient à l'aspect externe de l'individu, notamment) sont interdits, ce que révèlent les exemples suivants :

(3) Kakoj Vanja ? - (??Tëmnovolosyj + ??vysokij). [Comment est Vanja ? - Il est (châtain + grand)].

Cette interdiction est cependant levée lorsque la question se construit à l'aide d'expressions du type $s$ vidu. La question se traduit alors par Comment est-il de sa personne?

(4) Kakoj on s vidu? - (Tëmnovolosyj + vysokij). [Comment est-il de sa personne ? - Il est (châtain + grand).]

Les exemples cités illustraient les prédicats qui sélectionnent un sujet $1+$ humain/. Si le sujet est exprimé par un inanimé concret, l'interrogation en kakoj s'avère impossible ${ }^{8}$ :

(5) Kakaja mašina? - *Krasivaja. *Belaja ${ }^{9}$ [Comment est la voiture ? - Elle est belle. Elle est blanche.]

Les exemples cités mettent en valeur les différences de fonctionnement des pronoms interrogatifs dans les deux langues, sans pour autant remettre en question la division des états en hyperclasses. Nous le verrons dans la suite de ce paragraphe.

Le deuxième grand ensemble que nous évoquerons regroupe les prédicats d'états transitoires. Ils répondent à la question Dans quel état est-il ? et en russe, V kakom sostojanii ? Un grand nombre de classes sont concernées: les états

8. L'interrogation en какой demande une réponse du type «identification» : Kakaja u vas mašina? - «Lada». [Quelle voiture avez-vous ? - Une «Lada»]. Cette contrainte est levée lorsque la propriété exprimée par le prédicat est vue comme inhérente dans le contexte de l'énonciation. Ainsi, s'agissant d'un jeu au cours duquel la mère parle à l'enfant en lui montrant des images d'un livre : Kakoje solnce ? - Žëltoje. [Comment est le soleil ? - Il est jaune.]

9. Les questions appropriées seraient ici Mašina krasivaja? [La voiture est jolie ?] et Kakogo cveta mašina? [De quelle couleur est la voiture ?], respectivement. 
physiques généraux des objets et des humains, les états de veille ou de sommeil, les états de fonctionnement, etc. Les prédicats mentionnés ont ceci de commun qu'ils caractérisent leur référent à un moment donné de son existence. Par exemple:

(6a) Dans quel état est ta voiture? - Elle est en bon état de marche.

(6b) V kakom sostojanii ( $u$ vas) mašina ? - Ona slomana. [Dans quel état est (votre) voiture ? - Elle est cassée.]

(6c) Dans quel état est ton frère ? - Il est (abattu + malade).

(6d) V kakom sostojanii bol'noj ? - On (v kome + bez soznanija $)^{10}$.

[Dans quel état est le malade ? - Il est (dans le coma + inconscient).]

Les prédicats qui figurent dans ces exemples décrivent aussi leur référent. Dès lors, ils peuvent, dans une certaine mesure, être rapprochés des propriétés. Or, justement, l'application du paramètre de l'interrogation révèle les limites de cette analogie : les prédicats d'états transitoires impliquent un laps de temps. L'état en question est vrai pendant une période donnée.

Les deux hyperclasses citées (propriétés, états transitoires) ne représentent pas la totalité des adjectifs d'états. D'après nos observations, un nombre relativement important de prédicats sont interrogeables par Est-ce que ? en français. Quant au russe, il s'agira d'interrogation par l'intonation (qui ne possède pas de marqueur lexical spécifique). Par exemple:

(7a) Est-ce que cette réponse est correcte ?-Oui/Non.

(7b) Eto pravil'nyj otvet ? - Dal Net. [Cette réponse estelle correcte? - Oui/ Non.]

Comme on peut le constater, les prédicats qui entrent dans cette catégorie renvoient à un état de choses qui se prête à l'affirmation ou à la négation: connu, coupable, enceinte, etc.

10. Il est à noter cependant qu'en russe, ce type d'interrogation peut difficilement viser un être humain conscient et actif. Le substantif bol'noj [malade] constitue, à ce titre, une exception, ce qui peut-être dû au fait que bol'noj dénote un sujet plutôt passif, inactif. 
en français et izvestnyj [connu], vinoven [coupable], beremenna [enceinte], etc. en russe. De ce fait, ils forment un ensemble à part entière, non encore décrit par les chercheurs. Ils occupent une place toute particulière parmi les adjectifs d'états car ils n'acceptent aucun autre type d'interrogation. En effet, ils ne désignent pas leur référent et ne le décrivent pas non plus, il ne s'agit pas ici de propriétés perceptibles de l'objet auquel ils se rapportent.

Grâce au paramètre de l'interrogation, nous avons pu distinguer les propriétés et les états transitoires, deux hyperclasses possédant chacune ses spécificités. Nous avons aussi révélé l'existence de prédicats qui se situent en dehors de cette dichotomie et qui demandent à ce titre une étude particulière. Les différences entre le russe et le français concernent quelques classes isolées.

\section{L'intensité}

Le paramètre de l'intensité possède une portée double. Il va nous permettre, d'une part, de diviser les états en ceux qui sont «intensifiables» (qui acceptent divers marqueurs de degré), et ceux qui ne le sont pas. Il va aussi révéler, parmi ce premier ensemble, que divers prédicats exigent des marqueurs d'intensité spécifiques. Nous réunissons ci-dessous les éléments qui le prouvent.

Les prédicats qui acceptent les marqueurs de degré sont traditionnellement désignés par le terme de «scalaires $^{11}$ » ou "gradables». Leur particularité tient au fait que l'état [au sens large] qu'ils décrivent permet des degrés correspondant à diverses positions sur l'échelle sémantique. Ces prédicats sont illustrés par les exemples suivants :

(8a) L'homme était très grand et maigre, un peu voûté. (Guy de Maupassant, Le tic)

(8b) Pasynkov byl očen' vežliv i krotok so vsemi (I. Turgenev, Jakov Pasynkov). [Pasynkov fut très poli et affable avec tout le monde].

11. J.-C. Anscombre et O. Ducrot utilisent les termes «gradation » et «scalaire» (Anscombre, Ducrot, 1978: 46). Le terme «scalaire» est aussi utilisé dans R. Rivara (1993 : 40-46). 
Les prédicats d'aspect physique et de comportement, qui figurent dans ces exemples, sont intensifiables. De ce fait, les marqueurs d'intensité (très, un peu en français et очень en russe) sont naturels, ils traduisent le haut ou le faible degré de l'état. On observe la situation inverse dans les exemples suivants:

(9a) *L'homme était très mort.

(9b) *Čelovek byl očen' živ. [*L'homme était très vivant].

Ces prédicats expriment un état qui ne permet pas de degrés. L'idée d'intensité véhiculée par le marqueur est en contradiction avec le sémantisme du prédicat. Il s'agit de prédicats qui forment des séries et traduisent des états opposés. Lorsque l'un des éléments de la série est employé, les autres sont automatiquement exclus. Le même phénomène de blocage s'observe dans l'exemple suivant, même si la raison en est différente :

(10a) *Le dernier livre de cet auteur est très superbe.

(10b) *Etot roman - očen' potrjasajuščij. [*Ce roman est très superbe].

Pour L. Picabia (1978:15), ces prédicats sont des sortes de superlatifs. L'idée de haut degré est déjà présente dans le prédicat. De ce fait, les marqueurs lexicaux se révèlent superflus et les phrases ne sont pas naturelles.

Après avoir illustré l'opposition entre les prédicats scalaires et non-scalaires, nous nous pencherons sur les marqueurs d'intensité propres à ces premiers. Dire d'un prédicat d'état qu'il est scalaire ne suffit pas pour prédire de façon exhaustive son comportement face aux marqueurs d'intensité. D'après nos observations, chaque classe possède ses propres marqueurs ${ }^{12}$. Les prédicats de propriétés (illustrés par les exemples ci-dessus) acceptent les adverbes et les locutions adverbiales qui dénotent le haut ou le faible degré. Cela est aussi vrai des états transitoires:

(11a) Les enfants étaient très fatigués.

(11b) Pridvornye damy byli očen' ogorčeny tem, čto ne uvidjat moskovskogo prazdnestva (B. Akunin,

12. Il n'entre pas dans notre propos de décrire la totalité des marqueurs possibles, mais plutôt d'éclairer quelques tendances générales. 
Koronacija). [Les dames de la cour furent très chagrinées de ne pas pouvoir assister aux festivités moscovites].

Le haut degré d'intensité de l'état physique fatigués et psychologique ogorčeny est traduit ici par très et par son équivalent russe očen [très].

Pour terminer ce paragraphe consacré à l'intensité, nous voudrions mentionner quelques prédicats qui se situent, en quelque sorte, hors dichotomie «scalaire - non-scalaire». Ils n'acceptent pas les marqueurs de degré, mais permettent néanmoins ceux de complétude (Kupferman, 1991: 56). Par exemple :

(12a) La place de Grève était à moitié vide. (A. Mathiez,

La Révolution française).

(12b) Malen'kij zapotevščij grafin byl napolovinu pust.

(M. Aldanov, Begstvo) [La petite carafe embuée était à moitié vide].

Le prédicat fait partie d'une série (vide - plein, etc. pust [vide] - zapolnen [rempli], etc.). Entre deux pôles, des états intermédiaires, des «phases» sont possibles. Pour les traduire, la langue a recours aux locutions adverbiales du type $\grave{a}$ moitié, aux trois quarts, en français et na tri četverti [aux trois quarts], napolovinu [à moitié] en russe. Si nous avons évoqué ce phénomène, c'est parce qu'on peut faire le même constat avec d'autres prédicats qui ont en position de sujet un inanimé concret ou un locatif. Parmi eux, ouvert - fermé, otkryt [ouvert] - priotkryt [entr'ouvert] - zakryt [fermé], etc. Ils méritent à ce titre une étude particulière.

Le paramètre de l'intensité est doublement important, d'une part pour isoler les prédicats scalaires et de l'autre, pour décrire les marqueurs d'intensité spécifiques à chaque classe. C'est le sémantisme du prédicat qui explique qu'il accepte les marqueurs de degré. Mentionné par divers auteurs, ce paramètre n'a cependant pas encore été analysé dans sa complexité.

\section{Le temps et l'aspect}

Nous allons présenter ces paramètres ensemble dans la mesure où ils sont en synergie. D'après nos observations, la 
majorité des états se combine avec les marqueurs de temps et d'aspect. Par exemple:

(13a) Hier, je me sentais trop délabré, et aujourd'hui je veux, je veux à toute force travailler. (G. Flaubert, Correspondance).

(13b) Včera on byl neujazvimyj, ť̌česlavnyj, silnyj. (L.Bud'akova, Možno, ja umru vmeste s toboj). [Hier, il était invulnérable, vaniteux, fort].

Les prédicats d'état physique (délabrê) et de propriété (neujazvimyj, tščeslavnyj, silnyj) sont ici accompagnés d'adverbes temporels. Ceux-ci assument l'inscription de l'état dans le temps en le situant sur l'axe temporel ${ }^{13}$. Cette particularité a une conséquence non négligeable sur le fonctionnement du paramètre de l'aspect. En effet, les prédicats qui acceptent les marqueurs temporels permettent aussi les marqueurs d'aspect ${ }^{14}$ :

(14a) Pendant toute la soirée, il a été gai, causeur. (E.Legouvé, Soixante ans de souvenirs).

(14b) Ves' den' ona byla skučna. Naprasno Afanasij Ivanovič šutil i xotel uznat', otčego ona tak vdrug zagrustila: Pul'xerija Ivanovna byla bezotvetna (N. Gogol, Starosvetskije pomeščiki). [Pendant toute la journée, elle s'est ennuyée. C'est en vain qu'Afanasij Ivanovič plaisantait et essayait de savoir pourquoi elle était subitement devenue si triste. Pul'xerija Ivanovna ne donna aucune réponse.]

Dans ces phrases, les marqueurs de restriction temporelle (pendant toute la soirée, ves' den'), combinés à un prédicat d'état psychologique (gai, skučna), permettent de préciser la durée de l'existence de l'état. Lorsque le prédicat accepte ce type de marqueurs, nous dirons, suite à G. Gross, qu'il relève de l'aspect «interne» duratif.

Nous opposerons l'aspect interne à l' aspect «externe ${ }^{15} \gg$ en distinguant l'inchoatif, le continuatif et le terminatif selon

13. L'information temporelle est aussi apportée ici par les verbes supports qui, au même titre que les adverbes, jouent le rôle de marqueurs.

14. Notre classification des valeurs aspectuelles est conforme à (G. Gross, 1996a).

15. Conformément à G. Gross (1999-2000), l'aspect «interne» est l'aspect fondamental du prédicat, qui lui est propre par son sémantisme. 
qu'ils mettent l'accent sur le début, la continuation ou la fin de l'état exprimé par le prédicat ${ }^{16}$. Ainsi, s'agissant des prédicats de propriétés, la valeur aspectuelle inchoative peut être traduite par les supports devenir et se faire (en français) et stat' [devenir] et sdelat'sja [se faire] (en russe):

(15a) Il est devenu gros.

(15b) Ona sdelalas' vspylčiva, želčna, brančiva.

(F. Dostoevskij, Netočka Nezvanov) [Elle était devenue irascible, bilieuse, acariâtre].

Le prédicat du premier exemple renvoie à l'aspect physique, alors que dans le second, il s'agit d'un trait de caractère. On constate que les marqueurs cités traduisent le changement d'état du sujet humain et le passage vers l'état gros et vspylčiva [irascible]. A ce niveau, un fait important doit être signalé. En français, le changement d'état accompli est rendu par le passé composé du verbe devenir. En russe, en revanche, l'aspect perfectif ${ }^{17}$ du verbe sdelat'sja est en interaction avec l'aspect lexical. Autrement dit, l'analyse de la phrase russe doit tenir compte de l'interaction avec les deux types d'aspect, morphologique et lexical. Ce même constat est aussi vrai quant il s'agit de l'aspect terminatif. En français, la fin d'un état est souvent rendue par l'auxiliaire cesser de et en russe, par perestat' [cesser de]. Par exemple:

(16a) Il a cessé d'être gentil.

(16b) Kogda k nemu prišlo vseobščee priznanije - kak na rodine, tak $i$ za rubežom - on [Landau] perestal byt' zadiristym. (Ju. Rumer, Stranički vospominanij o L.D. Landau) [Lorsqu'il devint mondialement connu, aussi bien dans sa patrie qu'à l'étranger, il cessa d'être belliqueux].

L'aspect «externe», en revanche, regroupe les valeurs aspectuelles qui $s$ 'ajoutent à la valeur essentielle durative ou ponctuelle du prédicat.

16. Compte tenu des limites de cet article, certains prédicats seulement feront l'objet d'analyse ici.

17. Le verbe imperfectif correspondant n'est pourtant pas exclu dans ce contexte, par exemple: Ona delalas' vspylčiva, esli ne videla ego. [Elle devenait irascible si elle ne le voyait pas]. La phrase dénote alors un changement d'état répétitif. Il va de soi que l'exemple cité n'est qu'une illustration d'un phénomène complexe dont il est impossible de rendre compte entièrement dans le cadre de la présente étude. 
En combinaison avec les prédicats d'états (ici, prédicats de comportement, gentil et zadiristyj), les marqueurs cités traduisent la valeur terminative. En russe, l'auxiliaire aspectuel perestat' [cesser de] relève de l'aspect perfectif et exprime la fin de l'état zadiristyj [belliqueux].

Jusqu'à présent, nous n'avons cité que les marqueurs aspectuels du type verbal (supports et auxiliaires). Mais on ne peut pas passer sous silence le fait que très souvent, diverses valeurs aspectuelles sont prises en charge par des adverbiaux. Ainsi, pour exprimer la continuation d'un état, le russe peut avoir recours à la locution adverbiale vsë ešče [encore]. Nous allons l'illustrer par un prédicat d'état transitoire:

(17) Ja vpervye v žizni usnul sidja. Sonnyj ja upal. I kogda sosluživcy menja podnjali, ja vsë ešče byl v lëgkom obmoroke (A. Makanin, Odin i odna). [Pour la première fois de ma vie, je m'endormis assis. Et dans mon sommeil, je tombai. Lorsque les collègues me relevèrent, j'étais encore légèrement inconscient].

Par l'emploi de vsë eščë, le locuteur insiste sur la persistance de l'état exprimé par le prédicat de <conscience> v lëgkom obmoroke. D'une façon analogue, en français, cette valeur aspectuelle est prise en charge par l'adverbe encore (continuatif). Par exemple:

(18) Le Sud-Coréen pris en otage en Irak par des islamistes était encore en vie, lundi 21 juin 2004 au soir $<w w w$.internet-general.info>, site consulté le 02-04-07).

Il s'agit ici d'un prédicat de la classe des < états de vie ou de mort > et l'adverbe encore induit que l'état du sujet humain (le Sud-Coréen) reste stable, inchangé.

Ces quelques exemples nous ont permis d'illustrer les principes de description des prédicats d'états du point de vue de l'aspect. Nous revenons à présent au constat fait au début de ce paragraphe concernant l'hétérogénéité des états. Si la plupart acceptent les marqueurs de temps et d'aspect, cela n'est pas vrai de la totalité de ces prédicats. Certaines classes ne vérifient aucune des deux conditions mentionnées. Les prédicats qui relèvent de ce type sont notamment ceux qui renvoient à des caractéristiques qui sont propres à leur sujet (humain ou objet) tant que celui-ci existe. Par exemple: 
(19a) La Terre est ronde.

(19b) Zemlja kruglaja. [La Terre est ronde].

Le prédicat ronde (kruglaja) renvoie à une caractéristique intrinsèque de l'objet la Terre (Zemlja) et de ce fait, elle ne peut pas être «enlevée». Il s'agit d'un état qui existe sans la mention du temps et le prédicat n'accepte pas de marqueurs temporels, par exemple:

(20a) * La Terre a été ronde hier.

(20b) *VČera Zemlja byla kruglaja. [Hier, la Terre était ronde].

Ce premier constat est lié à un second constat selon lequel les adverbes et locutions adverbiales de durée ne peuvent pas se combiner avec ces prédicats. Les exemples le confirment :

(21a) * La Terre a été ronde pendant trois jours.

(21b) *Zemlja byla kruglaja tri dnja. [La Terre a été ronde pendant trois jours].

Le blocage est ici d'ordre sémantique. La valeur de la locution adverbiale est en contradiction avec celle du prédicat. Nous rangeons ces prédicats dans la catégorie des propriétés définitionnelles. Les caractéristiques auxquelles ils renvoient sont vraies tant que l'objet existe, ainsi être rond est une propriété intrinsèque de la Terre. L'incompatibilité entre ces prédicats et les marqueurs temporels (comme hier) ou aspectuels est d'ordre sémantique. L'état considéré renvoie à une situation dont on ne peut pas prendre un instantané. Ces quelques remarques concernant les propriétés définitionnelles sont capitales pour la raison suivante : les états sont traditionnellement associés à la notion de durée. Le cas des prédicats comme rond/NO: Terre constitue, à ce titre, une exception notable. En effet, l'état décrit par le prédicat est de nature durable, or les marqueurs d'aspect interne s'avèrent impossibles.

L'application des paramètres «temps » et «aspect» a permis d'observer quelques règles générales. La majorité des états accepte les uns aussi bien que les autres. Ces marqueurs peuvent varier suivant la classe sémantique à laquelle appartient le prédicat. Enfin, il existe des prédicats qui, bien qu'ils dénotent un état des choses durable, sont de nature atemporelle et de ce fait, interdisent tout marqueur temporel et aspectuel. 
Dans cet article, nous avons tenu à montrer que 1'analyse des adjectifs d'états doit tenir compte de plusieurs paramètres. Ils révèlent chacun le caractère sémantique profond des prédicats. L'ensemble des paramètres, dont certains n'ont encore jamais été abordés, permet de décrire chaque classe de façon systématique en vue d'établir une typologie (propriétés, états transitoires, etc.). Ils mettent en évidence les liens entre la classe sémantique et les propriétés combinatoires et transformationnelles des lexèmes qui en font partie.

La comparaison du russe et du français, deux langues de groupes différents, a révélé qu'il s'agit de paramètres de portée générale. En russe comme en français, les prédicats se prêtent à 1 'analyse en termes d'interrogation, de temps, d'aspect et d'intensité. Elles divergent cependant sur un certain nombre de points. Le premier concerne le paramère de l'interrogation, et en particulier, le fonctionnement en russe du pronom-adjectif kakoj ? et de son équivalent français comment? Le deuxième point relevé par cette étude est d'ordre grammatical et il tient au fait qu'en russe, au niveau d'une phrase, l'aspect lexical interfère avec l'aspect morphologique (perfectif-imperfectif). Cependant, nous avons essayé de démontrer que malgré ces points qui opposent les deux langues, la validité des paramètres d'analyse proposés n'est pas remise en question et ladite méthode pourrait être utilisée pour décrire tous les états (prédicats adjectivaux, nominaux ou verbaux). 


\section{BIBLIOGRAPHIE}

Akmalova, F.Š. (2005): Semantičeskaja i formal'no-strukturnaja reprezentacija kategorii «sostojanije» (na materiale anglijskogo, nemeckogo i russkogo jazykov), Avtoreferat kandidatskoj dissertacii, Iževsk.

ANscombre, J.-C. et DuCRoT, O. (1983): L'argumentation dans la langue, Liège, Pierre Madraga Éditeur.

BARTNING, I. (1985) : Aspects syntaxiques et sémantiques des adjectifs ethniques, Revue romane, 19-2, 177-217.

BULYGINA, T.V. (1982): K postrojeniju tipologii predikatov v russkom jazyke, Semantičeskije tipy predikatov, Moskva, Nauka, 7-85.

GRoss, G. (1991): Typologie des adjectivaux, Analyse et synthèse dans les langues romanes et slaves, $V^{e}$ Colloque international de linguistique slavo-romane, Bad Homburg, 9-11 Octobre 1989, Tübingen, G. Narr, 163-178.

Gross, G. (1996a): Prédicats nominaux et compatibilité aspectuelle, Langages, 121, 54-73.

Gross, G. (1996b): Les expressions figées en français : noms composés et autres locutions, Paris, Ophrys.

Gross, G. (1999-2000): Cours de DEA.

HARRIS, Z.S. (1976): Notes du cours de syntaxe, Paris, Seuil.

KoKochKINA, I. (2004): Typologie des prédicats d'états, Thèse de doctorat sous la direction de Gaston Gross, Université Paris XIII.

KUPFERMAN, L. (1991): Structure évènementielle de l'alternance un/0 devant les noms humains attributs, Langages, 102, 52-75.

LAKOFF, G. (1966): Stative Adjectives and Verbs in English, Mathematic linguistics and automatic translation, Report $N^{\circ} 17$, Cambridge, Harvard Computational Laboratory, 116.

Le Goffic, P. (1993): Grammaire de la phrase française, Paris, Hachette.

Miller, J.E. (1970) : Stative verbs in Russian, Foundations of Language, 6, 488-504.

PADUCEVA, E.V. (1996): Semantičeskije issledovanija. Semantika vremeni $i$ vida $v$ russkom jazyke, Moskva, Jazyki russkoj kul'tury. 
RIEGEL, M. (1985) : L'adjectif attribut, Paris, PUF.

Riegel, M., Pellat, J.-C. ET Rioul, R. (1994), Grammaire méthodique du français, Paris, Quadrige/PUF.

RivarA, R. (1993): Adjectifs et structures sémantiques scalaires, L'Information grammaticale, 58, 40-46.

TESNIERE, L. (1959): Éléments de syntaxe structurale, Paris, Kliencksieck.

VALGINA, N.S.: Sintaksis sovremennogo russkogo jazyka, <http://www.hi-edu.ru/ebooks/xbook089/01/index.html?part-014.htm> (page consultée le 30-04-07)

VENDLER, Z. (1967): Linguistics in Philosophy, Cornell University Press, Ithaca, NY, 97-121.

Wilmet, M. (2003): Grammaire critique du français, Bruxelles, Duculot. 